\title{
Riccardo Viel
}

\section{La lirica tra Provenza e Toscana}

\section{Contatti di culture e tradizioni manoscritte nel XIII e XIV secolo}

\begin{abstract}
Il saggio indaga la circolazione in area toscana dei canzonieri provenzali, facendo il punto sulle tre ondate di immissione di materiale trobadorico in Toscana e sulla sua ricezione. Tali elementi portano alla fine del Duecento alla definizione di un canone dei trovatori oltremodo originale tanto culturalmente quanto politicamente: il ceto mercantile rivestì infatti un ruolo fondamentale per una nuova declinazione dell'ideologia trobadorica e per un certo tipo di aggregazione dei testi, con la decisiva conseguenza che in Toscana, a differenza di quanto accade nelle corti settentrionali, non si ebbe una produzione in provenzale.

This contribution discusses the circulation of Provençal songbooks in medieval Tuscany, with special focus on the three "waves" of troubadour literature arriving in Tuscany and its reception. In the late thirteenth century, this led to an original definition of the canon of troubadours, both in cultural and political terms. Merchants played a particularly important role in the shaping of a new troubadour ideology and a certain type of text collections. As a consequence, Tuscany never witnessed any text production in Provençal, differently from what happened at the courts in northern Italy.
\end{abstract}

Parole chiave: Provençal songbooks, Troubadours, Merchants

Il quadro che dovrò qui delineare, ossia la circolazione in area toscana dei canzonieri trobadorici nel XIII e XIV secolo, è oltremodo complesso, oltreché indagato a fondo negli ultimi decenni da molti validi studiosi. È per questo che il mio intervento in questa sede si limiterà a una messa a punto di quanto sta efficacemente affiorando nei recenti scavi, nella quale non rinuncerò tuttavia ad azzardare qualche spunto di riflessione personale e a organizzare la lettura delle dinamiche ricostruttive da un punto di vista specifico, giungendo ad alcune inferenze finali che vorrei quantomeno tentare di sussumere da questo quadro in un più ampio contesto storico e socio-culturale.

Mi sarà inevitabile, ciò di cui mi scuso chiedendo indulgenza al lettore, incorrere in semplificazioni e inevitabili rinvii bibliografici a studî dedicati ad

Riccardo Viel, Università degli Studi di Bari Aldo Moro

Ә Open Access. (C) 2021 Riccardo Viel, published by De Gruyter. (c) BY This work is licensed under the Creative Commons Attribution 4.0 International License.

https://doi.org/10.1515/9783110702231-003 
aspetti tecnici ai quali dovrò accennare ma di cui non potrò approfondire i dettagli.

Inizierei presentando brevemente i canzonieri provenzali che descrivono la ricezione della poesia trobadorica nella Toscana del Due e Trecento. Si tratta del canzoniere J, confezionato tra XIII e XIV secolo in area linguadociana, assemblato con materiali provenienti dalla Francia settentrionale, e poi entrato in Toscana probabilmente sulla via dei mercanti fiorentini, come dimostrato recentemente da Cesare Mascitelli; ${ }^{1}$ il che illumina un aspetto interessantissimo, dal punto di vista sociale, del fenomeno di ricezione di questa letteratura nella realtà comunale, su cui tornerò tra poco. Quindi $\mathrm{P}$, coevo di J, confezionato a Gubbio ma probabilmente composto di materiale anche fiorentino, come attesta la patina linguistica del glossario provenzale-italiano che vi è contenuto dopo la sezione dei testi lirici. Poi F, esemplato nel XIV secolo non sappiamo se in Italia settentrionale o centrale, ma senz'altro legato a $\mathrm{P}$ da forti vincoli strutturali ed ecdotici, come $\mathrm{S}$, esemplato all'inizio del Trecento.

Ancora U, di mano fiorentina, trascritto tra XIII e XIV secolo, esemplare di pregio e di grande importanza come vedremo per il canone toscano dei trovatori.

Vi è quindi $\mathrm{V}^{2}$, sezione di mano trecentesca toscana del canzoniere $\mathrm{V}$ esemplato in Catalogna, e poi giunto in Italia centrale. Infine c, codice umanistico del XV secolo assai prossimo a U.

Tra tutti questi, il canzoniere $\mathrm{P}$ appare, insieme ad U, il perno della circolazione dei materiali trobadorici in Toscana, fotografando una situazione che si sedimenta attorno agli anni 60-90 del Duecento.

La circolazione del materiale trobadorico in Toscana sembra però essere ben più antica. Stefano Resconi ipotizza una prima immissione organizzata del materiale trobadorico agli anni Venti del XIII secolo. ${ }^{2}$ Nel canzoniere Q, infatti, si reperisce una pastorella anonima (Quant eu escavalcai l'autr'an, BdT 461,200), da mettere in relazione con una cobla di Paves conservata nel canzoniere H (Anc de Roland ni del pro N'Auliver, BdT 320,1) che mette in scena un aspro diverbio tra giullari, occorso a Firenze. Se il primo componimento sembra topograficamente doversi allocare alla zona delle Alpi Apuane, il secondo è da ricondurre, sulla scorta di Gianfranco Folena, alla così detta «accademia tabernaria», gravitante attorno alla corte estense. Resconi mette quindi in relazione questi dati alla stratigrafia linguistica della sezione Q3 del canzoniere $\mathrm{Q}$, che secondo Ilaria Zamuner presenta tratti linguistici toscani

1 Mascitelli, Il canzoniere trobadorico J.

2 Resconi, Note sulla sezione; anche Resconi, La lirica trobadorica. 
sovrapposti a una patina settentrionale, arrivando a delineare un asse Este corte dei Malaspina - Firenze attivo nel terzo decennio del Duecento.

Si tenga presente che tale datazione appare molto alta, pressoché a ridosso della sedimentazione del materiale trobadorico della tradizione *epsilon*, come la chiamerà Avalle. ${ }^{3}$ Perdonate la semplificazione che devo ora compiere per descrivere rapidamente tale costellazione ecdotica. Secondo molti - non entro nel merito delle possibili confutazioni di questa tesi, pure importanti - il trovatore Uc de Saint Circ sarebbe approdato alla corte di Alberico Da Romano, signore di Treviso e trovatore in provenzale, fratello del Vicario imperiale Ezzelino. Uc avrebbe dunque esemplato per lui una raccolta della poesia trobadorica, il cosiddetto (e perduto) Liber Alberici. Tale raccolta, già secondo Gröber, la cui fonte è grosso modo la fonte chiamata *beta* da Avalle, si sarebbe fusa con altri materiali, dando luogo a una grande 'editio variorum' che Avalle definisce 'collettore epsilon'. Sempre semplificando, in questo scriptorium d'area veneta si compie la compilazione del grande canzoniere estense D, databile al 1254, il quale molto probabilmente non è l'esito diretto della fusione delle due fonti ${ }^{\star}$ beta $^{\star}$ più ${ }^{\star}$ epsilon ${ }^{\star} ;{ }^{4}$ la creazione del Liber Alberici è dunque da porre tra il 1230 e prima della metà del secolo (1240, forse). Ma su questi passaggi stemmaticamente alti le ipotesi sono molte; da quelle di Zufferey, a quelle di Meliga e Lachin, su cui non ho tempo di soffermarmi. ${ }^{5}$

Quello che ci interessa è che questo collettore costituisce un vero e proprio canone, letterariamente vigilato e trascelto, strutturalmente organizzato, che s'imporrà rapidamente come punto di riferimento per tutto o quasi il milieu poetico trobadorico dell'Italia padana, imperandovi sino alle sue propaggini trecentesche. La genesi e la trasformazione di questo canone, nei suoi varî prodotti (canzonieri $\mathrm{A}, \mathrm{B}, \mathrm{I}, \mathrm{K}, \mathrm{N}$, ecc.) è nota e non mi ci soffermo. Ma richiamo il suggestivo studio di Zinelli dove efficacemente si riconducono gli unica di IK a un ambiente più propriamente veneto lagunare con un'interessante fusione con la tradizione linguistica della letteratura francese d'oltremare, su cui tornerò più avanti. ${ }^{6}$

Insomma, il ‘canone' di *epsilon* è letterariamente vincente. Eppure da più parti emergono tracce di tradizioni orientali 'non epsilon', tra cui i frammenti $\mathrm{z}$

\footnotetext{
3 Avalle, I manoscritti della letteratura in lingua d'oc.

4 Si veda Zinelli, Il canzoniere estense.

5 Zufferey, Genèse et structure; i volumi I trovatori nel Veneto e a Venezia e Il medioevo nella Marca e i saggi ivi contenuti; inoltre almeno Meliga, I canzonieri trobadorici I e K; Meneghetti, Il pubblico dei trovatori; Zinelli, Sur les traces; Zinelli, Il canzoniere estense.

6 Zinelli, Sur le traces.
} 
ed $\mathrm{m}$, accordi in lezione di manoscritti come $\mathrm{Na}$, fonte che ho chiamato ${ }^{\star} \mathrm{ni}^{\star}{ }^{7}{ }^{7} \mathrm{e}$ gli stessi unica di IK a cui facevo riferimento prima richiamando il saggio di Zinelli. Tracce che a noi appaiono tali oggi, perché probabilmente il grande canone *epsilon* ha nel tempo spazzato via ciò che da esso esorbitava. Ma non possiamo non considerare l'eventualità che altri materiali organizzati si fossero sedimentati ad esempio attorno all'influenza della corte estense.

A proposito di questo tema abbiamo ora la messa a punto di Luca Gatti, che attende senz'altro una verifica nell'intricata foresta ecdotica della filologia dei canzonieri, ma che già da ora sembra suggerire l'emersione di un qualche ruolo del canzoniere $\mathrm{N}^{8}{ }^{8}$

Desta un certo interesse, dunque, che dagli studi di Resconi affiori che materiale estense, incentrato sulle tenzoni rissose tra Mola e Peire de la Mula, potesse in qualche modo già tra 1220 e 1230 essere penetrato in area toscana, passando per la corte dei Malaspina, sedimentandosi precocemente nel canzoniere Q (a proposito del quale, sia detto per inciso, Zinelli rammenta concordanze in errore proprio con $\mathrm{D}^{\mathrm{a}}$, cioè forse il materiale del Liber Alberici).

Torniamo però senz'altro alla Toscana. Dopo questa prima 'ondata' di immissione di materiale trobadorico, sempre Stefano Resconi periodizza un secondo afflusso, probabilmente più cospicuo, attorno al 1260-70. Tale afflusso appare evidente studiando il suo prodotto maggiore, appunto $\mathrm{P}$, soprattutto nella sua prima sezione e nelle citazioni liriche della sezione biografica. Lo studioso, mettendo a profitto anche precedenti studi di Maria Luisa Meneghetti, ${ }^{9}$ rinviene nuovamente un percorso da Nord verso Sud attraverso gli Appennini, di fatto una sorta di riattivazione dello stesso alveo produttivo nella prima metà del Secolo. Insomma, un'attrazione della corte dei Malaspina con Genova e Monferrato sullo sfondo. Questa volta però i materiali sarebbero per la gran parte di provenienza da un collettore attivo negli anni Quaranta nel bacino padano nord-occidentale.

Le conferme vengono dalla prima sezione del manoscritto $P$, che è funestata da sorprendenti errori attributivi, dando a Blacasset e a Giraut de Borneil testi sicuramente di paternità diversa.

Decisiva, in particolare, è l'assegnazione di Be.m platz a Blacasset. Resconi nota come tale errore attributivo, che si riverbera anche in U e V2, altri prodotti connessi all'area toscana, e che dunque deve trovarsi a monte della costituzione del canzoniere $\mathrm{P}$, potesse essersi creato, come suggeriva Beltrami, ${ }^{10}$ già in Provenza alla corte di Blacatz, di cui Blacasset era figlio. L'alternanza

\footnotetext{
7 Viel, Sulle tracce.

8 Gatti, I trovatori alla corte estense.

9 Meneghetti, Lancelot, Guenièvre e Rigaut de Berbezilh; Meneghetti, Uc e gli altri.

10 Beltrami, Remarques sur Guilhem de Saint Gregori.
} 
Blacatz/Blacasset è frequente nei prodotti di area padana, ${ }^{11}$ che la derivano da una ricezione dunque già viziata in partenza, alla corte di Aups: Resconi nota come sulla figura di Blacasset l'estensore della vida (certamente italianosettentrionale e veneto) riversi quella che Meneghetti chiamava una «struttura encomiastica» ${ }^{12}$ riservata al ben più famoso e potente padre. ${ }^{13}$ Tutto ciò porta a ritenere come in area italiano settentrionale si fosse sedimentata una sostanziale sovrapposizione tra le figure di Blacatz e Blacasset, in un milieu di poeti che aveva eletto $B e \cdot m$ platz a canzone-manifesto, per motivi soprattutto politici. Proprio lì si sarà raccolto il materiale, estraneo ad *epsilon*, poi unito in una gelegenheitsammlungen, una raccolta di fogli sparsi e disorganizzati, poi passati verso Sud tramite il Monferrato e la corte di Malaspina, sino ad organizzarsi nella prima parte di $\mathrm{P}$, generando gli errori attributivi a tutti noti. A riprova, Resconi identifica con chiarezza ed efficacia una trama di rimandi tra un componimento adespoto, Honraz es home per despendre, con testi di Lanfran Cigala e Luchetto Gattilusio, in particolare Be.m meravill del marques Moruel, che chiama in causa Moroello Malaspina. Si crea così un legame tra l'area genovese, la corte dei Malaspina e del Monferrato, e l'area padana. Tutto ciò a conferma delle intuizioni di Stefano Asperti che già nel 1995 riteneva fondamentale la figura di Federico Malaspina per la seconda parte del canzoniere P, in Nuls hom non deu d'amic ni de segnor. ${ }^{14}$

Insomma, il materiale su cui verrà organizzato in area Toscana ciò da cui deriva la prima parte di $\mathrm{P}$ - ma, abbiamo visto, anche la terza parte delle coblas esparsas - si sposta dall'Italia settentrionale a sud degli Appennini, attraverso il Monferrato e la corte dei Malaspina, anche rinnovellando l'asse, che abbiamo visto attivo già nella prima ondata, con l'ambiente estense, come suggeriva Stefano Asperti notando contatti e allusioni testuali, ricorrenze di autori, tra diverse parti della sezione di coblas esparsas del canzoniere P con il florilegio del canzoniere F di Ferrarino da Ferrara e alcune sezioni del canzoniere H. Tale materiale ritrova forse una prima significativa sosta nel genovese. Si sposta poi verso Est passando per Pisa. Ma dell'asse Genova-Pisa parlerò tra poco.

A conferma di quanto qui esposto, anzi diremmo di quanto sistematizzato da Resconi, vanno gli studî un poco più recenti di Marco Grimaldi (siamo nel

11 Oltre a quanto in Asperti, Carlo I d'Angiò e i trovatori, p. 172 e nell'edizione critica Der Troubadour Blacassetz, su questo segnalo il lavoro della mia allieva Barbara Francioni, la cui tesi di laurea dal titolo Il trovatore Blacasset, nuova edizione critica del poeta, è stata discussa presso l'Università di Bari nel 2019.

12 Meneghetti, Il pubblico dei trovatori, p. 242.

13 Resconi, Note sulla sezione, p. 226.

14 Asperti, Carlo I d'Angiò e i trovatori, pp. 179-181, 186 sgg. 
2011) a proposito del canzoniere a, il noto canzoniere di Bernart Amoros. ${ }^{15}$ Efficacemente lo studioso ricostruisce la possibilità che un grappolo di tenzoni politiche, databili tra 1260 e 1270, di carattere ghibellino e anti-angioino, sia stato agglutinato al canzoniere di Bernart Amoros nel suo percorso da Nord verso l'area genovese. Questi Liederblätter che, sono d'accordo con Grimaldi, probabilmente sedimentavano autonomamente e poi venivano sistematizzati nei canzonieri, testimoniano un interesse di questo 'canone' alle vicende imperiali del milieu post federiciano. Ci tornerò in conclusione.

Non sfugge a Resconi un rapporto stretto tra P, e dunque il primo canone trobadorico toscano, e la produzione quasi contemporanea provenzale. Si tratta di un dato evidente, rappresentato dall'ampia sezione dedicata in $\mathrm{P}$ a Bertran Carbonel, trovatore marsigliese, e già anticipata dalla tenzone tra Granet e Bertran de Lamanon, come aveva già notato nel 1995 Stefano Asperti. Quest'ampia sezione accomuna, peraltro, $\mathrm{P}$ al codice $\mathrm{R}$. Resconi ritiene si celi qui un rapporto biunivoco tra l'ambiente toscano e quello provenzale. E poi un rapporto con la Catalogna, attraverso la mediazione dell'asse genovese-pisano sul quale avevo promesso di tornare, grazie alla centralità di un personaggio come Terramagnino da Pisa, attraverso cui la Toscana conosce le Razos de Trobar sulle quali egli compone la Doctrina d'Acort.

Si diceva dell'asse Genova-Pisa: da tempo Fabrizio Cigni lavora con risultati eccellenti di sintesi alla messa a punto di questa direttrice di circolazione testuale. ${ }^{16}$ In tal senso un altro tassello importante è, come ha mostrato recentemente lo studioso, la presenza del frammento di canzoniere provenzale p. Esso si rivela indubbiamente legato all'ambiente genovese, e lì assemblato probabilmente negli anni Novanta del Duecento; ma nella sua stratigrafia linguistica compaiono elementi di patina veneto-orientale che lasciano pensare a una raccolta di materiale proveniente da quell'area padana. Ciò fa sistema con numerosi indizî ecdotici che accomunano p a S. Nello stesso $\mathrm{S}$, come già aveva notato Asperti, è peraltro densissima la presenza di oitanismi nei testi trobadorici tramandati, ciò che lascia pensare che il materiale della loro fonte comune, e dunque anche comune a $\mathrm{P}$ che per tanti versi è affine a $\mathrm{S}$, derivasse in buona misura dall'area veneta.

Proprio il rapporto con l'area oitanica appare da valorizzare, anche nella tradizione toscana. Assai interessante è infatti il componimento Non sai qe je dire, tràdito da $\mathrm{P}$ nella sezione delle coblas esparsas e lì attribuito, erronea-

15 Grimaldi, La poesia storico-politica; per completezza anche: Grimaldi, Svevi e Angioini, che lega il discorso all'ambiente pisano-genovese.

16 Ad esempio cfr. Cigni, Manuscrits en français, italien, et latin; Cigni, Due nuove acquisizioni. Per il nostro discorso cfr. anche Cigni, In margine alla circolazione. 
mente, a Sordello. Asperti ha tratto decisive inferenze dal dato di tradizione: l'erroneità attributiva è riferita alla 'angioinità' della seconda produzione del poeta mantovano, e l'origine del mottetto (altrove musicato) è individuata in ambienti angioini di Montpellier e forse non distanti dalla produzione di Adam de la Halle. Ecco quindi che la componente angioina emerge con buone fondamenta nello studio del canzoniere laurenziano già dal 1995. Peraltro, nell'àmbito della sezione Q3 del canzoniere Q, cui accennavo poc'anzi a proposito della prima ondata di ricezione del materiale trobadorico toscano, occorre ricordare che la stessa Zamuner, analizzando il corpus delle baladas provenzali, riteneva possibile collegare quel materiale, posto nella fonte di Q3, all'ambiente di Carlo I d'Angiò. ${ }^{17}$ Il canale di contatto tra l'ambiente francese, quello padano, e l'asse toscano-genovese è dunque biunivoco: da Sud a Nord e da Nord a Sud. In tale contesto, non si può lasciare in ombra l'ultima sezione del codice $\mathrm{P}$, che dopo le liriche provenzali, un Donatz proensals e un glossario provenzale italiano con indubbia patinatura veneta, ci tramanda il poemetto misogino anticofrancese Le blasme de femmes, che secondo Pagano presenta forti indizî linguistici anglonormanni. Di questo testo si è recentemente occupato Davide Battagliola, ${ }^{18}$ studioso che ritiene di poter collegare la patina di questo reperto ad un francese veneziano, o comunque orientale. Un tassello in più, che potrebbe forse - ma attendiamo le indagini già avviate - portare a considerare questi lacerti oitanici non lontani da un'idea di scripta d'oltremare che si legherebbe bene all'ambiente pisano-genovese come a quello veneziano.

Non vorrei ora mettere altra carne al fuoco, il quadro essendo già abbastanza complesso, ma non posso non completarlo accennando al canzoniere U, il prodotto rappresentativo della fase ultima e terminale dell'organizzazione del materiale trobadorico in area toscana, diciamo il 'canone toscano' idealmente da guardare in contrasto al 'canone epsilon'. Dopo la 'prima ondata' di immissione di materiale trobadorico in Toscana testimoniato da alcuni brani di Q, la seconda ondata testimoniata dal canzoniere $\mathrm{P}$, ora il canone fiorentino dei trovatori si completa alla fine del XIII secolo con U.

Se il canzoniere $\mathrm{P}$ rappresenta la sedimentazione toscana di un canone trobadorico prevalentemente contemporaneo, ossia rivolto alla produzione dei trovatori del XIII secolo, soprattutto coinvolti nelle dinamiche socio-culturali italiane, U recupera e organizza il canone fiorentino dei trovatori dell'epoca aurea, ossia del XII secolo - aprendosi solo nell'ultima sezione alla contemporaneità. Nella prima sezione del canzoniere abbiamo infatti un elenco disposto

17 Zamuner, Le «baladas» del canzoniere provenzale $Q$.

18 Battagliola, Processo alle donne. 
per ordine d'importanza decrescente: Giraut de Bornelh, Arnaut Daniel, Folchetto di Marsiglia, Aimeric de Pegulhan, Gaucelm Faidit, Cadenet, ecc. sino a Jaufre Rudel e Raimbaut d'Aurenga per ultimi. È il canone del gusto postsiciliano, sul quale si sarà formato il primo gusto trobadorico di Dante e l'idea del fenomeno provenzale che traluce nella cultura letteraria comunale (come nel Novellino, la novella di Rigaut, come il perdigonismo di Chiaro Davanzati, il bernardismo di Bondie Dietaiuti, l'arnaldismo di Pucciandone Martelli). Tutti materiali che provengono (semplifico molto) da centri di riscrittura stemmaticamente piuttosto bassi, dell'Italia settentrionale, di ambiente *epsilon* e non-^epsilon*. Importante è ad esempio una fonte ${ }^{\star}$ lambda* attiva in epoca tarda, che Resconi individua; ricordo che il canzoniere L, principale prodotto di *lambda*, è stato recentemente ricondotto ad ambienti mercanteschi da Sabina Marinetti, come si è visto di J. ${ }^{19}$

Nella seconda parte, meno strutturata, confluiscono innesti seriori che integrano questo 'gusto siciliano', come lo chiama Resconi, ${ }^{20}$ con la poesia politica e la tenzonistica più recente, riattivando quei canali che avevamo visto presenti già in P. Infatti ecco l'aggiunta della sezione di Bertran de Born, testi che rappresentano particolari declinazioni della fin'amor e componimenti dialogici, e unica che vanno ricondotti a un ambiente ostile a Bonifacio di Monferrato, databile tra il 1238 e il 1245, plausibilmente di area genovese.

Insomma, si può dire che il dittico P-U ci testimonia l'idea e il paradigma dei trovatori che doveva essersi sedimentato a Firenze nel Duecento.

Vediamo ora come questo quadro di circolazione testuale possa coniugarsi al dato storico-letterario e culturale.

1) Un primo dato che si può evincere è che la Toscana non è affatto al di fuori del fenomeno di ricezione della poesia trobadorica. Non esiste, dunque, solo ${ }^{\star}$ epsilon ${ }^{\star}$. E probabilmente quella che Avalle riteneva essere la Terza Tradizione - ora non entro nella spinosa questione della sussistenza ecdotica di tale etichetta in quanto famiglia, su cui nutro gli stessi dubbî di Luca Barbieri e Stefano Resconi ${ }^{21}$ - è probabilmente un complesso fenomeno di costituzione di un canone, dunque di revisione di materiali, che coinvolge PSUc ma anche Q, in certa misura T, F, H e J. Insomma, la Toscana organizza e recepisce, in una fase alta, dato che la prima 'ondata' è allocabile al 1220, il materiale trobadorico, arricchendosi sia da fonti che troviamo gravitare attorno all'area estense, sia da fonti di area nord-occidentale, sia di area provenzale, sia di area catalana, sia infine forse di materiale oitanico veicolato dal milieu angioino e da contatti tra l'area pisano-genovese e gli ambienti d'oltremare.

19 Marinetti, Il canzoniere provenzale L e Venezia.

20 Resconi, La lirica trobadorica, p. 293; ma vedi anche le pp. 316-322.

21 Barbieri, «Tertium non datur»?; ma cfr. Le canzoni di Uc de Saint-Circ. Saggio di edizione critica; Zinelli, À propos d'une édition récente; Zinelli, La chanson Be fai granda follor. 
Un secondo dato è che la costituzione e organizzazione di questo canone presenta indubbiamente tratti originali, sia culturalmente sia politicamente. Non è satellite della scuola trobadorica genovese; né è epigonica rispetto al canone padano *epsilon*.

2) L'idea che progressivamente si affermi una declinazione dell'ideologia trobadorica attraverso cui si esprime un ceto di podestà comunali e notai/magistrati itineranti è già stata ben documentata da contribuiti non recentissimi di Stefano Asperti, ${ }^{22}$ di Armando Antonelli, ${ }^{23}$ e di altri, ed è una chiave di lettura proficua per comprendere la sedimentazione di alcuni 'grappoli' di componimenti, tenzoni, attorno ai Malaspina, nel Monferrato, con un significativo rapporto con le realtà comunali emergenti ad Arles e nella valle del Rodano. Ovviamente uno degli epicentri è Firenze, e il quadro diventa oggi sistematico. Qui, al rapporto tra Arles e il milieu genovese, per il tramite di Percivalle Doria, ricordato da Montefusco, ${ }^{24}$ aggiungerei la presenza di Raimon Bistorz d'Arle in F, da ricondurre all'ambiente estense, e direttamente coinvolto in $\mathrm{P}$ almeno per Ara agues eu do(m)pna uostra beutatz.

L'emersione del miles quale cavaliere 'cittadino' è forse all'origine di quelle gelegenheitssamlungen che precocemente si radunano attorno a sirventesi come $B e \cdot m$ platz, o a tenzoni rissose come quelle ricondotte all'ambiente dell'accademia tabernaria estense.

C'è poi il ruolo giocato, in tutto ciò, dal ceto dei ricchi mercanti. Ricordo qui lo studio della sezione terminale del canzoniere $\mathrm{P}$, con quel poemetto oitanico analizzato da Battagliola, accennato poc'anzi. Battagliola avvicina alla versione del poemetto conservata in $\mathrm{P}$ un'altra versione della stessa opera conservata nel Pluteo 76. 79, di copista forse bolognese; entrambi questi testimoni essendo stati probabilmente al séguito di esponenti dell'emergente ceto mercantizio. Questo è un indizio importante per legare questi prodotti e questo paradigma culturale a un ambiente - quello dei mercanti fiorentini - che già emergeva a proposito del canzoniere J, e ora di L, come accennavo all'inizio. Un ambiente non estraneo, peraltro, come avevo già cercato di dimostrare in un mio contributo sul Fiore pronunciato alla Società Dantesca qualche anno fa, ${ }^{25}$ allo stesso poemetto attribuibile a Dante, il cui parafraste mostra, nel lessico, di adottare oitanismi spiegabili all'interno del vocabolario di mercanti fiorentini frequentatori delle fiere d'Oltralpe. Oitanismi che, nel Fiore, convivono con provenzalismi lirici, e oitanismi letterarî, al punto che il profilo del parafraste sembrerebbe ben collimare con - ad esempio - una figura quale il compilatore del canzoniere J o del canzoniere P: un colto mercante. L'interferenza tra manufatti differenti, l'interferenza

22 Asperti, Carlo I d'Angiò e i trovatori, in particolare pp. 186-200.

23 Antonelli, Pedrini, Appunti sulla formazione.

24 Montefusco, Livelli di cultura.

25 Viel, Oltre la traccia, in particolare pp. 112-114 e sgg. 
tra lingue e generi letterarî all'interno degli stessi manufatti, lo stesso carattere composito di questi manoscritti induce a ritenere che il ceto mercantesco sia uno dei fattori di scambio e di contatto culturale in questa fase e all'interno dell'ambiente comunale.

Abbastanza conseguente a questo panorama è il quadro descritto sempre da Montefusco con la discesa di Carlo d'Angiò, e l'emersione di una nuova saldatura tra filo-angioini e ambienti di grandi investitori del ceto bancario comunale. ${ }^{26}$ Concordo che un siffatto quadro politico tra anni Sessanta e Settanta del Duecento possa essere alla base della produzione poetica che si coagula attorno a personaggi quali Raimon de Tors, Bertran d'Alamano, Luchetto Gattilusio ecc., poeti implicati in costellazioni di canzonieri quali $\mathrm{H}, \mathrm{M}, \mathrm{T}$, f, e infine $\mathrm{P}$. Il canone toscano come appare in $\mathrm{P}$, e come si sedimenta nella Toscana durante la seconda ondata d'immissione di materiale trobadorico, in quel torno d'anni, riflette certamente questo quadro intellettuale e politico. In effetti tali considerazioni si accordano in buona misura con le conclusioni più eminentemente filologiche del quadro ricostruttivo prodotto in questi anni da Asperti e Resconi da un lato, Cigni e dal gruppo Guiron dall'altro per il panorama pisano-genovese. ${ }^{27}$ Così come all'interno di questi processi si potrebbe forse intravedere un aggancio, se mai c'è stato, tra Luchetto Gattilusio e Guido Guinizzelli a Bologna, innervando sull'asse Genova-Pisa-Firenze anche l'asse Bologna-Firenze. Non saprei. Osservo però che, a differenza dell'ambiente Veneto di *epsilon* e dell'ambiente genovese, in terra toscana la formazione e progressiva organizzazione di questo canone trobadorico non dà luogo a una produzione trobadorica locale, di cui rimangono gli sparuti e un po' deprimenti esperimenti dei sonetti in provenzale di Terramagnino e di Dante da Maiano, Paolo Lanfranchi di Pistoia o le coblas anonime gravitanti attorno alla figura di Nino Visconti, per cui si veda da ultimo Giorgio Barachini (ma in tal caso s'insiste più che altro sull'asse genovese-pisano -gallurese). ${ }^{28}$

Insomma, in Toscana, e segnatamente a Firenze, sembra che rimanga ben chiara la refrattarietà alla lingua altra, ossia all'uso di volgari diversi, quand'anche al centro di questi processi vi fosse, come certamente v'era, la figura di Brunetto Latini, egli stesso rifacitore del suo Trésor nella versione

26 Montefusco, Livelli di cultura.

27 Il Gruppo Guiron, che opera presso la Fondazione Ezio Franceschini, è diretto da Lino Leonardi e Richard Trachsler, coordinato da Nicola Morato, e vanta la collaborazione di Luca Cadioli, Fabrizio Cigni, Claudio Lagomarsini, Sophie Lecomte, Ilaria Molteni, Francesco Montorsi, Noëlle-Christine Rebichon, Anne Schoysman, Elena Stefanelli, Marco Veneziale, Barbara Wahlen, Fabio Zinelli.

28 Barachini, La lotta delle "partes". 
fiorentina "abrégé" del Tesoretto. ${ }^{29}$ Significativamente il canzoniere $\mathrm{P}$, che è stato interpretato come una sorta di 'avviamento agli studi provenzali' ante litteram, contiene il canone poetico trobadorico espressione del contesto politico-culturale che si è detto, un glossario che traduce dal provenzale al toscano, e due manuali di retorica e poetica: il Donatz proensals e le Razos de Trobar. Eppure, sebbene con tutto il necessario per conoscere quella lirica, tale canone è essenzialmente poi utilizzato per tradurla in una lirica prettamente toscana e specialmente fiorentina, non per comporre e riproporre una poesia in provenzale.

Se si può trarre qualche conclusione, forse si potrà osservare una differenza di fondo tra l'ambiente toscano e gli altri ambienti settentrionali esaminati. Sembra tutta toscana infatti questa attitudine alla ricezione e organizzazione dell'esperienza lirica trobadorica anche e soprattutto contemporanea, questa riduzione a canone a cui però non segue una replicazione quasi osmotica tra ricezione provenzale e ricreazione in provenzale, come accade invece nelle corti del Nord e nel genovese, per tutto il Duecento e ancora nel XIV secolo.

Il canone toscano sembra dunque polarizzare un interesse per gli 'eventi politico-letterari' che stabiliscono una continuità evolutiva con le vicende postfedericiane. Lo si è visto nella sezione finale del canzoniere di Bernart Amoros, in chiave anti-angioina; ma lo si vede anche nella scelta dei brani tenzonistici di ambientazione non cortese di P, e dall'ultima sezione di U, quella dedicata ai trovatori contemporanei. Già la Magna Curia aveva tradotto, con una translatio di forme e di lingua, il canone trobadorico che aveva recepito; e siamo anche qui tra 1220 e 1230 . Quasi contemporaneamente comincia a sedimentarsi un 'canone' toscano testimoniato da alcuni componimenti emersi in Q. Poco più tardi, tra 1260 e 1270, come testimonia il canzoniere P, si cristallizza il primo canone formale toscano dei trovatori, poi completato da U. Non a caso ciò avviene quando l'esperienza poetica federiciana viene sistematizzata nei tre grandi canzonieri - Vaticano, Palatino e Laurenziano - sui quali richiamo gli studi di Lino Leonardi, e riproposta in chiave toscanizzata, dunque ancora translata. E allo stesso modo, e specularmente, viene translata la tradizione trobadorica in toscano. Non nasce una lirica trobadorica in Toscana.

Può darsi che vi sia una consapevolezza, in questi ambienti in contatto e in questi ceti emergenti (notai, figure dell'alta classe dei banchieri mercantizî, alti poteri comunali e podestarili), dell'importanza della translatio di quel paradigma nella lingua del comune (consapevolezza astrattamente parallela al dise-

29 Trésor di cui, peraltro, fu precocemente prodotto un volgarizzamento toscano, per lungo tempo attribuito - ed è significativo - a Bono Giamboni. 
gno del funzionariato curiale federiciano, benché non teorizzata come quello). Una consapevolezza forse maggiore di quanto avvenisse negli ambienti cortesi e post-cortesi delle corti del Nord, o nell'ambiente genovese, forse tutto sommato più intimamente plurilingui di quello tosco-fiorentino.

In questo senso mi pare che Dante sia un figlio del suo ambiente, di certo il più dotato (come prima di lui spicca la figura di Brunetto). Il De vulgari eloquentia è anche - non certamente solo, ma anche - un dettagliato canone letterario, linguistico, metrico, stilistico, retorico. Una ricezione che si fa riflessione, che si nutre del canone letterario delineato nel canzoniere $\mathrm{U}$, che si nutre forse di conoscenze linguistiche acquisite attraverso la lettura delle Razos de trobar, del Donatz proensals, desunte da canzonieri del tipo di $\mathrm{P}$, e poi delle progressive conoscenze di altri canzonieri nella primissima fase dell'esilio. Dante però apre al plurilinguismo, accoglie nel suo lessico elementi esogeni, e in certo senso con la Commedia rompe il canone, formalmente e linguisticamente. La translatio diviene refundatio, più compiutamente e consciamente di quanto già non fosse in quell'etichetta, tutta dantesca appunto, di Stil novo e di nove rime. Nella Commedia egli non si limita a superare la poesia provenzale, siciliana, siculo-toscana e stilnovista solo nell'aspetto letterario e contenutistico. Il superamento è precipuamente linguistico giacché, con una palinodia profonda del lessico topico lirico, egli rifonda semanticamente quei provenzalismi che erano percolati dal canone trobadorico all'interno della poesia siciliana e poi toscana. E tale rifondazione avviene parallelamente e contemporaneamente alla rifondazione del lessico mediolatino.

Lo snodo principale, che apre al secondo Trecento, è qui. Certo, Brunetto e Dante sono dei vertici; al di sotto di loro figure non meno interessanti, ma certo più sbiadite, avranno avuto ben inferiore consapevolezza di questi processi, e mi chiedo, pertanto, se sia lecito evincere un paradigma dal vertice applicandolo poi alla vasta platea che insiste al di sotto di queste "avanguardie". Ma non voglio addentrarmi oltre, perché ancora molto c'è da indagare circa le radici sociali e culturali di questo passaggio, a cui ho accennato, dalla translatio alla refundatio nell'ambiente toscano e fiorentino. 